\title{
Detecting Suicidal Ideation on Tweets
}

\author{
Vinícius de Carvalho Cardoso ${ }^{1}$, Antonio Fhillipi M. Silva ${ }^{2}$, \\ Roberta Akemi Sinoara $^{3,4}$, Solange Oliveira Rezende ${ }^{3}$, Dario Brito Calçada ${ }^{1,3}$ \\ ${ }^{1}$ Universidade Estadual do Piauí (UESPI) \\ Campus Alexandre Alves de Oliveira - Parnaíba, PI - Brazil \\ ${ }^{2}$ Universidade Estadual do Piauí (UESPI) \\ Campus Dra. Josefina Demes - Floriano, PI - Brazil \\ ${ }^{3}$ Instituto de Ciências Matemáticas e de Computação (ICMC) \\ Universidade de São Paulo (USP) - São Carlos, SP - Brazil \\ ${ }^{4}$ Instituto Federal de Educação, Ciência e Tecnologia de São Paulo (IFSP) \\ Campus Boituva - Boituva, SP - Brazil \\ \{viniciuscc94, dariobcalcada\}@gmail.com
}

\begin{abstract}
According to the World Health Organization, every 40 seconds, one person dies of suicide in the world. Among young people aged from 15 to 29, suicide is the second leading cause of death. Still, these deaths can be prevented. In this scenario, social networks like Twitter can become real-time sources of information and help to prevent suicide. This paper presents an initial exploration of the problem of identifying individuals at risk of self-extermination in social networks that use Portuguese language. As a main scientific contribution, a set of tweet data, manually labeled by experts, was built and can be used for future research on the subject. As a preliminary evaluation, we applied machine learning algorithms for classification. The results indicate that the dataset can be used in a study to develop a real-time suicidal ideation tweet detection system.
\end{abstract}

\section{Introdução}

Segundo a OMS (Organização Mundial da Saúde), a cada 40s uma pessoa morre por suicídio no mundo. É estimado que, só em 2012, 804.000 suicídios tenham ocorrido, representando uma média anual global de 11,4 a cada 100.000 pessoas. Entre jovens de 15 a 29 anos, o suicídio é a segunda maior causa de mortes globalmente. Porém, esses números podem ser ainda maiores, uma vez que, em muitas ocasiões, devido a uma classificação equivocada como acidente ou outra causa de morte, ou, ainda, em países onde o ato é ilegal, casos de suicídio não são reportados [OMS 2014].

Apesar desses números preocupantes, mortes por suicídio podem ser prevenidas [Bailey et al. 2011]. Nesse cenário, a detecção precoce de risco de suicídio pode prover a base para programas de intervenção, o que pode ser efetivo em prevenir tais mortes. E, embora pessoas suicidas não se sintam motivadas a revelar seus pensamentos ou planos antes de uma tentativa, o período que precede um suicídio pode conter indícios a respeito da intenção do indivíduo [OMS 2014].

Diante de uma realidade de indícios, métodos tradicionais de prevenção não conseguem identificar pessoas em risco de suicídio em tempo real [McCarthy 2010]. 
Nesse cenário, redes sociais a exemplo do Twitter, que é uma plataforma de expressão pessoal, podem se tornar fontes de informação em tempo real e ajudar na prevenção do suicídio [Jashinsky et al. 2014]. De fato, pesquisas têm mostrado que indivíduos em risco estão recorrendo a tecnologias contemporâneas (fóruns, micro-blogs) para expressar seus problemas sem precisar encarar alguém pessoalmente [Moreno et al. 2011, De Choudhury et al. 2013]. Inclusive, casos de vítimas de suicídio escrevendo seus pensamentos finais nessas comunidades online já foram reportados [Gunn and Lester 2015, Kailasam and Samuels 2015]. Não obstante, muitos trabalhos têm encontrado relação entre risco de suicídio e padrões linguísticos em posts das redes sociais [McCarthy 2010, Sueki 2015].

Apesar de não haver um consenso entre diferentes comunidades de pesquisa [Miner et al. 2012], a Mineração de Textos (MT) pode ser vista como a aplicação de um conjunto de técnicas usadas para analisar dados não estruturados e descobrir padrões que não eram conhecidos previamente [Aggarwal and Zhai 2012]. Assim, a MT pode ser tratada como uma especialização da Mineração de Dados. Enquanto a Mineração de Dados trata os dados estruturados, a MT lida com textos escritos em língua natural (dados não estruturados). Com o crescente aumento e variedade de documentos textuais, tanto em redes sociais e Web em geral quanto internamente em organizações, as técnicas de MT têm se tornado essenciais no apoio à descoberta de conhecimento. Com isso, as fontes de textos, bem como as aplicações da MT, são variadas.

De forma geral, o processo de MT pode ser visto como um processo formado por cinco etapas, conforme ilustrado na Figura 1. Esse processo se inicia com a especificação de seus objetivos na etapa de Identificação do Problema. Nesta etapa, o analista, especialista em MT, deve delimitar o escopo da mineração, preferivelmente trabalhando com um especialista do domínio de aplicação. Devem ser definidas as coleções de textos que serão mineradas e como os resultados serão utilizados. As especificações definidas na etapa de Identificação do Problema guiarão as próximas etapas do processo de MT, as quais podem ser executadas em ciclos de preparação dos dados (etapa de Pré-processamento), descoberta de conhecimento (etapa de Extração de Padrões) e avaliação do conhecimento (etapa de Pós-processamento) [Sinoara et al. 2017].

Visando reduzir o número de termos e amenizar os problemas da alta dimensionalidade e esparsidade, pode-se utilizar algumas técnicas de pré-processamento, como:

- Remoção de stopwords: a remoção de stopwords visa a eliminação de palavras que não trazem informação relevante para o processo de MT. Essas palavras, chamadas de stopwords, normalmente são palavras que possuem as funções de artigos, preposições, pronomes e conjunções. No entanto, também podem ser identificadas stopwords específicas do domínio de aplicação do processo, ou seja, palavras que sabidamente são frequentes na coleção e que não distinguem classes ou grupos que espera-se identificar com a Mineração de Textos.

- Normalização: a normalização dos textos visa eliminar as diversas variações que as palavras podem sofrer, como por exemplo variações de gênero e número dos substantivos e conjugações dos verbos. A normalização pode ser realizada por meio de três técnicas: (i) radicalização (stemming), que reduz cada palavra ao seu radical (ou palavra raiz); (ii) lematização, que reduz cada palavra a seu lema (ou forma canônica), ou seja, verbos são reduzidos ao infinitivo, e substantivos 


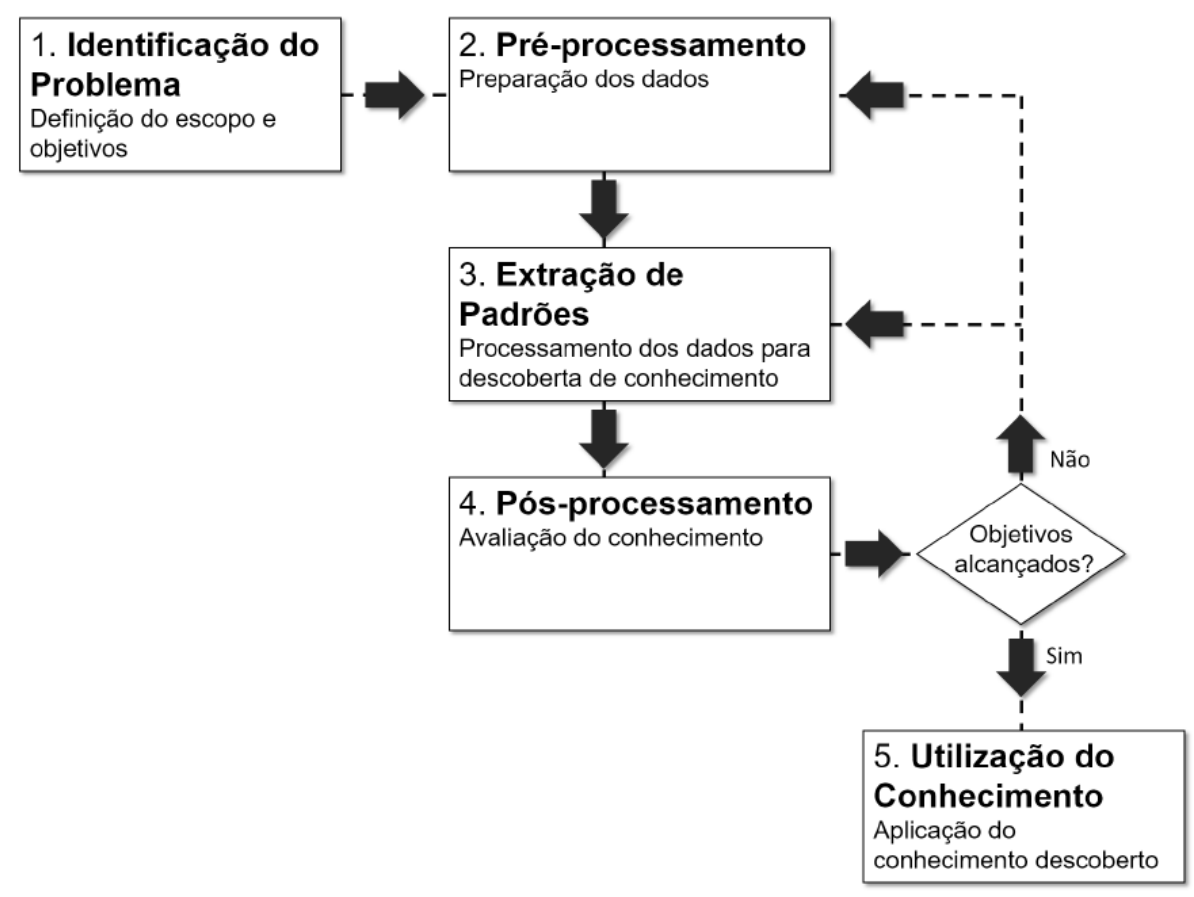

Figura 1. Processos de Mineração de Textos. Adaptado de [Sinoara et al. 2017]

e adjetivos são reduzidos a forma masculina singular; e (iii) substantivação, que transforma a palavra para que ela tenha o comportamento sintático/semântico semelhante a de um substantivo.

A busca pela detecção de ideação suicida em tempo real se apresenta como uma alternativa viável no auxílio a órgãos que cuidam deste tipo de autoextermínio. Este estudo visa preencher as lacunas na literatura em relação à análise e à classificação de conteúdo relacionado ao suicídio em Língua Portuguesa. Os principais objetivos deste trabalho são: i) criar um dataset público composto por tweets escritos em português para futuras pesquisas acerca do tema, e ii) comparar o desempenho de diferentes algoritmos de classificação de textos na distinção de tweets com e sem ideação suicida.

Os resultados foram promissores já que foi construído um dataset bem amplo de tweets classificados como sendo de ideação suicida ou não. O dataset foi rotulado por especialistas na área que validaram as respostas formando um conjunto de dados robusto e que poderá ser utilizado em outras pesquisas. Os algoritmos de classificação supervisionados apresentaram bom comportamento diante da complexidade dos dados apresentados.

Além dessa Seção introdutória o presente artigo é organizado de modo que na Seção 2 são apresentados as pesquisas que inspiraram este trabalho, bem como lacunas que ainda não haviam sido elucidadas. Na Seção 3 são apresentados os métodos utilizados para a coleta dos tweets e a rotulação dos dados, bem como os algoritmos utilizados na tarefa de classificação. Os resultados obtidos são apresentados na Seção 4. Por fim, na Seção 5 são apresentadas as considerações finais sobre a pesquisa, além de possibilidades de trabalhos futuros. 


\section{Trabalhos Relacionados}

Nos últimos anos, diversos trabalhos investigaram a possibilidade de criar modelos para a classificação de conteúdo relacionado ao suicídio nas redes sociais. Em [Abboute et al. 2014], foi descrito um processo completo para, automaticamente, coletar tweets suspeitos de acordo com um vocabulário de termos, criado pelos próprios autores, que pessoas suicidas costumam utilizar. Com a obtenção de um corpus que também incluía casos comprovados, os tweets foram classificados em "risky" e "non risky". Então o desempenho de seis classificadores foram comparados, obtendo uma acurácia de aproximadamente $64 \%$ para o algoritmo Nä̈ve Bayes.

Focados principalmente na criação do corpus, [Desmet and Hoste 2014] introduziram uma estratégia de anotação em cascata para reconhecer conteúdo suicida. Em tal esquema, os textos, obtidos do Netlog (rede social particularmente popular entre jovens na Holanda), foram analisados por aspectos como relevância, origem, sujeito, severidade e, ainda, se apresentavam fatores protetivos ou de risco para suicídio. Como melhor resultado obtido durante os experimentos, atingiu-se uma precisão de $79 \%$ com o algoritmo SVM (Support Vector Machines - Máquinas de Vetores de Suporte).

Dividindo os tweets em três classes, ao invés de apenas duas, [O'Dea et al. 2015] tinham como objetivo examinar se o nível de "preocupação" para um tweet relacionado ao suicídio poderia ser determinado baseando-se somente em seu conteúdo. Como principal resultado obtido, o classificador SVM classificou corretamente $80 \%$ dos tweets rotulados como "strongly concerning", i.e., extremamente preocupante, o que indica uma possível mensagem de uma pessoa com ideação suicida.

Em seu trabalho, [Burnap et al. 2017] foram mais a fundo. Primeiro, eles dividiram os tweets em mais classes (sete ao todo) para distinguir entre conteúdo mais preocupante (ex. ideação suicida) e outros também relacionados ao suicídio (ex. relatos de suicídio, campanhas preventivas, etc.). Além disso, na etapa de experimentos, os autores não só avaliaram o desempenho dos algoritmos individualmente, mas também construíram um ensemble desses classificadores, o qual levou aos melhores resultados obtidos.

Até a data de escrita deste artigo, nenhum trabalho com a mesma proposta dos acima citados foi encontrado em Língua Portuguesa. Com o intuito de preencher esta lacuna em nossa literatura, este trabalho visa realizar um estudo inicial na identificação de tweets, escritos em Português, que apresentam ideação suicida.

\section{Materiais e Métodos}

O trabalho está dividido em três principais etapas: i) coleta de dados, ii) rotulação manual por especialistas e iii) classificação com o uso de algoritmos de aprendizado de máquina.

\subsection{Coleta de Dados}

Como primeira etapa do processo de Mineração de Textos deve-se obter um conjunto de documentos pertencentes ao domínio do problema a ser resolvido. Assim, de 15 de março a 15 de abril, utilizou-se de uma API ${ }^{1}$ (Application Programming Interface - Interface de Programação de Aplicativos), disponibilizada pelo próprio Twitter, para automaticamente coletar um corpus de tweets que continham termos ou expressões próprios do vernáculo

\footnotetext{
${ }^{1}$ https://developer.twitter.com/en/docs/tweets/search/overview
} 
da ideação suicida [O'Dea et al. 2015]. Tais palavras e frases, por terem sido definidas em um trabalho em Língua Inglesa (ex. suicide, suicidal, end my life, not worth living), foram primeiramente traduzidas para o Português antes da realização da coleta. Na Tabela 1 são listadas todas as palavras e expressões selecionadas para captação dos tweets.

Tabela 1. Palavras e expressões do vernáculo da ideação suicida.

\begin{tabular}{|c|c|}
\hline Original & Tradução \\
\hline Suicidal & Suicida \\
\hline Suicide & Suicídio \\
\hline Kill myself & Me matar \\
\hline My suicide note & Meu bilhete suicida \\
\hline My suicide letter & Mcabar com a minha vida \\
\hline End my life & Nunca acordar \\
\hline Never wake up & Não consigo continuar \\
\hline Can't go on & Não vale a pena viver \\
\hline Not worth living & Pronto para pular; pronto pra pular \\
\hline Ready to jump & Quero morrer \\
\hline Sleep forever; go to sleep forever & Dormir para sempre; dormir pra sempre \\
\hline Want to die & Estar morto \\
\hline Be dead & Melhor sem mim \\
\hline Better off without me & Melhor morto \\
\hline Better off dead & Plano de suicídio \\
\hline Suicide plan & Cansado de viver \\
\hline Tired of living & Morrer sozinho \\
\hline Die alone & Não quero estar aqui \\
\hline Don't want to be here &
\end{tabular}

Com relação às palavras e expressões utilizadas para recuperar os tweets neste trabalho, algumas considerações devem ser feitas. Primeiro que, diferentemente do Inglês, os adjetivos sofrem flexão de gênero no Português. Optou-se, então, nos casos onde a expressão original tivesse adjetivo que o mesmo seria traduzido apenas para o masculino (ex. tired of living - cansado de viver). Outra decisão tomada durante essa tarefa foi, levando em consideração a natureza informal da escrita de tweets, traduzir alguns casos considerando tanto a norma padrão da língua quanto a informal (ex. sleep forever (dormir para sempre; dormir pra sempre)).

Embora a API do Twitter permita coletar vários dados a respeito de um tweet como o nome do usuário que o publicou, o número de retweets, local e data da publicação, etc., por questões que envolvem os objetivos deste trabalho, além das questões éticas, foram coletados e armazenados apenas o conteúdo textual dos tweets.

\subsection{Rotulação por Especialistas}

Para essa fase, uma amostra de 1190 tweets (aproximadamente $25 \%$ dos 4732 coletados) foi escolhida para anotação manual. Para a realização dessa tarefa, uma equipe de especialistas, composta por três psicólogos, foi formada. Os tweets foram organizados e tabulados a fim de que cada um dos especialistas pudesse realizar a leitura completa dos mesmos e responder a pergunta: "Esse tweet apresenta ideação suicida?". 
Os tweets da amostra apresentada puderam ser classificados em uma de três categorias possíveis: "positivo", "negativo" e "indefinido". A fim de ser rotulado como "positivo", o tweet deveria indicar ideação suicida por parte do usuário, o que poderia ser expresso por meio da presença de fatores de risco no texto. Desse modo, sempre que um tweet indicasse depressão ou outras desordens psicológicas, tentativas anteriores de suicídio, violência familiar, bullying, sentimentos de isolamento, impulsividade, entre outros fatores (American Foundation for Suicide Prevention [AFSP 2013]), receberia o rótulo "positivo".

Por outro lado, se um tweet apresentasse sarcasmo, fizesse referência a notícias ou, ainda, a campanhas de prevenção ao suicídio, o rótulo "negativo" deveria ser escolhido. Por fim, caso o especialista não conseguisse classificar o tweet como positivo ou negativo, ele poderia atribuir-lhe a categoria "indefinido".

Após a classificação manual realizada pelos 3 (três) especialistas, na qual cada um rotulou todos os tweets da amostra, sem nenhum tipo de contato com os outros dois, foi realizado um processo para selecionar os tweets que comporiam o dataset final a ser utilizado na etapa posterior.

Com a intenção de construir um dataset apenas com os tweets classificados como "positivo" e "negativo", todos os tweets rotulados de forma unânime como "indefinido" pela equipe de especialistas foram descartados. Todos os tweets que não receberam o mesmo rótulo por parte dos três especialistas também foram excluídos. $\mathrm{O}$ dataset final é composto por 699 instâncias e está disponível on-line no link http: //bit. ly/2SiogW7.

\subsection{Classificação utilizando algoritmos de aprendizado supervisionado}

Com os dados devidamente rotulados pela equipe de especialistas, algoritmos de aprendizado de máquina foram aplicados para derivar um classificador de texto capaz de predizer automaticamente a classe de cada tweet. A API do WEKA ${ }^{2}$ (Waikato Environment for Knowledge Analysis) foi utilizada para comparar o desempenho de um número de classificadores.

No entanto, como se trata da categorização de textos, técnicas de PLN (Processamento de Linguagem Natural) foram aplicadas, principalmente na etapa de préprocessamento dos tweets. A principal atividade realizada na etapa de Pré-processamento é a representação dos textos em um formato aceito pelo algoritmo a ser utilizado na Extração de Padrões. Os algoritmos tradicionais de Aprendizado de Máquina, que normalmente são utilizados na mineração de dados estruturados, assumem que os dados são apresentados em um formato conhecido como matriz atributo-valor. Nessa matriz, cada instância (ou exemplo) corresponde a uma linha e seus atributos (características que descrevem esse exemplo) correspondem às colunas.

Nos processos de MT, após a identificação do problema (Figura 1), é necessário o pré-processamento da informação. Nesta etapa busca-se realizar a mudança de um dado não estruturado (texto) para um estruturado. Desse modo, passos como tokenization, remoção de pontuação, remoção de stopwords e aplicação de stemming, comuns a qualquer pré-processamento de texto tradicional, foram realizados. Os tweets foram

\footnotetext{
${ }^{2} \mathrm{http}: / /$ www.cs.waikato.ac.nz/ml/weka/
} 
representados com a técnica de bag-of-words com a frequência simples de cada token presente.

Adicionalmente, outras técnicas especificamente aplicadas a tweets como remoção de referências a usuários e remoção de URLs, foram utilizadas [Go et al. 2009]. Assim, cada tweet foi transformado em vetor de $n$-grams (nesse caso, unigrams, ou seja cada palavra foi considerada como uma feature), seguindo a abordagem para representação de textos bag-of-words.

Neste trabalho foram aplicados sete algoritmos de classificação indutiva, tradicionais e estado da arte, sendo cinco algoritmos da biblioteca Weka [Witten and Frank 2005] e dois algoritmos baseados em redes bipartidas [Rossi et al. 2014, Rossi et al. 2016]. Apesar das representações avaliadas neste trabalho serem representações no modelo espaço-vetorial, a utilização de algoritmos baseados em redes bipartidas também é possível. As redes bipartidas podem ser obtidas por mapeamento direto de representações documento-termo, ou seja, representações no modelo espaço-vetorial [Rossi et al. 2016].

Com o objetivo de se avaliar as representações em cenários bem diversificados, buscou-se diferentes variações dos parâmetros utilizados para cada algoritmo. Os valores selecionados para os parâmetros dos algoritmos foram baseados nos valores utilizados na extensa avaliação experimental de classificação indutiva supervisionada realizada por [Rossi et al. 2016]. Assim, a seleção de parâmetros foi realizada visando a diversificação dos modelos e o uso de configurações utilizadas em outros trabalhos da literatura e que apresentam bons resultados. Os algoritmos e os parâmetros utilizados nas avaliações experimentais deste trabalho são apresentados a seguir.

- Naive Bayes (NB)

O algoritmo Naive Bayes é um classificador probabilístico baseado no "Teorema de Bayes", o qual foi criado por Thomas Bayes (1701 - 1761). Atualmente, o algoritmo se tornou popular na área de Aprendizado de Máquina para categorizar textos baseado na frequência das palavras usadas, podendo ser utilizado para identificação do assunto ao qual o texto se refere.

- Multinomial Naive Bayes (MNB)

O algoritmo MNB implementa o algoritmo Naive Bayes para dados distribuídos multinomialmente e é uma das duas variantes clássicas do NB usadas na classificação de texto (onde os dados são tipicamente representados como contagens vetoriais de palavras).

- $\mathrm{C} 4.5$

Foi utilizada a implementação da ferramenta Weka (J48). Foram utilizados os níveis de confiança 0,15, 0,20 e 0,25.

- Support Vector Machine (SVM)

Foi utilizado o algoritmo Sequential Minimal Optimization (SMO), implementação da ferramenta Weka. Foram utilizados três tipos de kernel: linear, polynomial (com expoente $=2$ ) e radial basis function. Os valores de $\mathrm{C}$ utilizados para cada tipo de kernel foram: $0,10^{-5}, 10^{-4}, 10^{-3}, 10^{-2}, 10^{-1}, 10^{0}$, $10^{1}, 10^{2}, 10^{3}, 10^{4}$ e $10^{5}$.

- k-Nearest Neighbors (k-NN)

Foi utilizada a implementação da ferramenta Weka (IBk). O algoritmo foi utilizado com e sem o voto ponderado pela distância entre os exemplos. Foram aplica- 


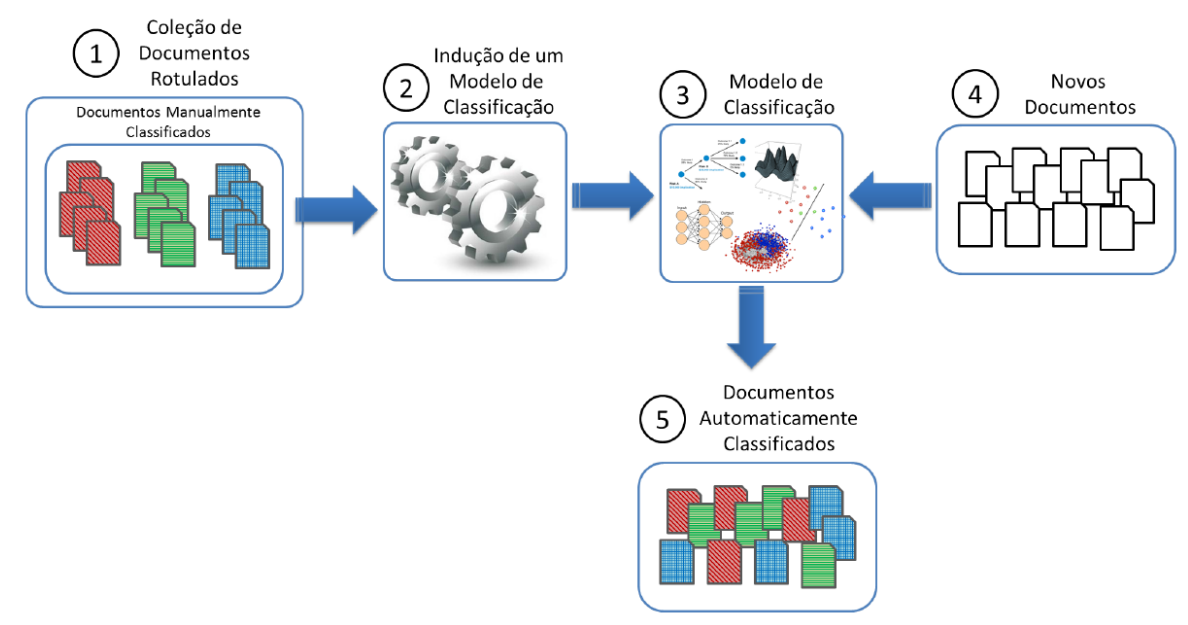

Figura 2. Esquema ilustrativo da classificação automática de textos por meio de aprendizado indutivo supervisionado. [Rossi 2016]

das duas medidas de distância: Cosseno e Euclidiana. Os valores de k utilizados foram: $1,3,5,7,9,11,13,15,17,19,25,35,45$ e 55 .

- $I M B H N^{C}$

Versão do algoritmo Inductive Model based on Bipartite Heterogeneous Networks, algoritmo baseado em redes heterogêneas bipartidas. Essa versão do algoritmo altera as informações de classe dos termos caso as informações de classe correntes produzam um erro de classificação. Foram utilizadas as taxas de correção de erro de $0,01,0,05,0,1$ e 0,5 . O número máximo de iterações foi definido em 1000 e o critério de parada foi definido como sendo o limiar de 0,01 para o erro quadrático médio.

- $I M B H N^{R}$

Versão do algoritmo Inductive Model based on Bipartite Heterogeneous Networks, algoritmo baseado em redes heterogêneas bipartidas. Essa versão do algoritmo realiza uma regressão para induzir as informações de classe dos termos. Foram utilizadas as taxas de correção de erro de $0,01,0,05,0,1$ e 0,5 . O número máximo de iterações foi definido em 1000 e o critério de parada foi definido como sendo o limiar de 0,01 para o erro quadrático médio.

Considerando-se o uso de algoritmos de aprendizado indutivo supervisionados, o problema de classificação automática de textos é definido como se segue. Dados um conjunto de classes $(C)$ e uma coleção de documentos rotulados $(D)$, documentos cuja classe é conhecida, um algoritmo indutivo supervisionado induz uma função $F$ que mapeia os documentos de $D$ a classes de $C(F: D \rightarrow C)$. A função $F$ é chamada de modelo de classificação (ou classificador) e é utilizada para predizer a classe de novos documentos. Esse processo é ilustrado na Figura 2.

A performance dos classificadores foram avaliadas por meio das medidas Acurácia (equivalente a Micro-F1), Precisão e Recall. As medidas foram obtidas por meio de 10-fold cross-validation. Todos os algoritmos foram avaliados segundo as mesmas partições dos dados no processo de treinamento e teste executados. As avaliações 
experimentais foram executadas por meio da ferramenta Text Categorization ${ }^{3}$, disponibilizada por Rossi e colabotadores [Rossi et al. 2016].

\section{Resultados e Discussão}

Um dos objetivos deste trabalho foi a construção de um dataset rotulado a ser utilizado em estudos acerca da detecção de ideação suicida em tweets. Assim, na seção 4.1, será descrito o processo de construção de tal dataset. Já na seção 4.2, é feita a comparação entre os resultados obtidos por cada algoritmo de Aprendizado de Máquina na tarefa de classificação de tweets.

\subsection{Dataset}

O dataset construído para este trabalho é composto por 699 instâncias. Cada instância representa um tweet rotulado manualmente e de forma unânime pela equipe de especialistas em "positivo" (211 instâncias) ou "negativo" (488 instâncias). Para ser classificado como "positivo", o tweet deveria apresentar indícios de ideação suicida por parte do usuário. Por outro lado, sempre que um tweet fizesse referência ao suicídio de terceiros, campanhas de prevenção ao suicídio, ou apresentasse sarcasmo e ironia, ele deveria ser classificado como "negativo".

Por fim, com os tweets rotulados, foi realizado o pré-processamento dos mesmos. Assim, etapas como tokenization, remoção de pontuação e caracteres especiais, remoção de stopwords e aplicação de stemming, usualmente presentes na Mineração de Textos tradicional, foram aplicadas. Adicionalmente, devido a características próprias dos tweets, como tamanho curto e o uso de linguagem informal por parte dos usuários, passos como remoção de referências a outros usuários e URLs, assim como a expansão de abreviaturas comuns na Internet foram realizados. Após todas essas etapas, obteve-se a configuração final do dataset, disponível on-line em http: / / bit. 1y/32aDPf 9.

\subsection{Classificação automática dos tweets}

Os experimentos de classificação foram realizados para validar o conjunto gerado. $\mathrm{Na}$ Tabela 2 são apresentados os melhores valores para acurácia obtidos para cada algoritmo em todas as configurações descritas na Seção anterior, juntamente com seus respectivos valores para Precisão e Recall. Observa-se que os desempenhos variaram bastante para todas as medidas. Para Acurácia, os valores vão de 30,18\%, obtido pelo algoritmo SVM em sua configuração Linear - 0, até $81,83 \%$ pelo mesmo algoritmo em sua configuração SVM (SMO) - RBFKernel - $10^{1}$, representando que o conjunto de dados permite uma sensibilização dos algoritmos de classificação. Portanto, o conjunto de dados gerado pode ser utilizado para elaboração de sistemas que utilizam técnicas de aprendizado de máquina a fim de detecção de tweets de ideação suicida em tempo real.

Comportamento similar é observado em relação às medidas de Precisão e Recall, nos quais os valores obtidos encontram-se no intervalo de 0,15 a 0,81 para Precisão e de 0,5 a 0,79 para Recall, mas sempre para configurações do algoritmo SVM (SMO), corroborando com o observado na literatura como o algoritmo que apresenta os melhores

\footnotetext{
${ }^{3}$ Text Categorization tool: http://sites.labic.icmc.usp.br/ragero/thesis/text_ categorization_tool
} 
resultados para a tarefa de classificação de textos, principalmente quando a abordagem bag-of-words para a representação dos textos é usada.

Nota-se também que os resultados alcançados neste artigo superam aqueles obtidos nos trabalhos relacionados citados anteriormente. Deve-se levar em consideração, obviamente, que tais trabalhos foram realizados para Língua Inglesa e sob configurações experimentais diferentes em relação a este. Ademais, por se tratar do primeiro estudo a abordar tal tema para textos em português, os resultados aqui obtidos poderão servir de baseline para futuros trabalhos.

Tabela 2. Resultados experimentais para Acurácia, Precisão e Recall.

\begin{tabular}{|c|c|c|c|}
\hline Algoritmo & Acurácia(\%) & Precisão & Recall \\
\hline NB & 80,11 & 0,76 & 0,74 \\
\hline MNB & 72,95 & 0,68 & 0,69 \\
\hline J48 - 0,25 & 78,39 & 0,75 & 0,74 \\
\hline SVM (SMO) - Linear - $10^{-1}$ & 80,97 & 0,79 & 0,73 \\
\hline SVM (SMO) - PolyKernel - $10^{-1}$ & 79,26 & 0,76 & $\mathbf{0 , 7 9}$ \\
\hline SVM (SMO) - RBFKernel - $10^{1}$ & $\mathbf{8 1 , 8 3}$ & 0,78 & 0,78 \\
\hline SVM (SMO) - RBFKernel - $10^{0}$ & 73,11 & $\mathbf{0 , 8 1}$ & 0,56 \\
\hline KNN Euclidiana K=1 & 60,95 & 0,69 & 0,70 \\
\hline KNN Coseno K=3 & 72,83 & 0,74 & 0,78 \\
\hline$I M B H N^{C}$ taxa 0,5 & 78,26 & 0,75 & 0,78 \\
\hline$I M B H N^{R}$ taxa 0,01 & 76,26 & 0,74 & 0,78 \\
\hline
\end{tabular}

\section{Conclusões}

Neste artigo, foi apresentado todo o processo de construção de um dataset formado com tweets de ideação suicida escritos em Língua Portuguesa. A não existência de um conjunto de dados para estudo desta temática em pesquisas de PLN motivou a execução deste trabalho, bem como pelo fato do número crescente de suicídios, que ocasiona um problema de saúde pública em vários países.

Para a construção do dataset, um corpus composto por tweets contendo palavras ou expressões condizentes com o vocabulário suicida foi coletado. Uma equipe de especialistas então foi encarregada de classificar manualmente os tweets, baseando-se apenas em seu conteúdo. No fim, após a realização do pré-processamento dos textos, obteve-se um dataset rotulado composto por tweets escritos em português e que está disponibilizado publicamente para futuras pesquisas no tema. A construção e a disponibilidade do dataset é a principal contribuição científica desta pesquisa.

Para validação do uso do dataset, foram comparados os desempenhos de um número de algoritmos de classificação para a tarefa de identificar conteúdo relacionado à ideação suicida no Twitter. A quantidade de algoritmos de classificação utilizados em variadas configurações possibilitou observar o comportamento destes algoritmos diante de um conjunto de dados coletado automaticamente do Twitter e rotulados manualmente por especialistas. A convergência de todos os algoritmos possibilitou a validação do uso do dataset construído, o que pode alavancar pesquisas nessa temática dentro da área de Mineração de Textos. 
Como trabalho futuro, poderá se utilizar ensembles de classificadores para medir o poder preditivo dos modelos de classificação. Outra melhoria possível seria a utilização de features que expressem as características emotivas e estruturais dos tweets. Por fim, outras abordagens, além da bag-of-words, para a representação dos textos também poderiam ser exploradas.

\section{Acknowledgment}

O presente trabalho foi realizado com apoio da Coordenação de Aperfeiçoamento de Pessoal de Nível Superior - Brasil (CAPES) - Código de Financiamento 001. Agradecimentos também aos especialistas psicólogos que participaram desta pesquisa e de todos aqueles que trabalham em prol da prevenção ao suicídio.

\section{Referências}

Abboute, A., Boudjeriou, Y., Entringer, G., Azé, J., Bringay, S., and Poncelet, P. (2014). Mining twitter for suicide prevention. In International Conference on Applications of Natural Language to Data Bases/Information Systems, pages 250-253. Springer.

AFSP (2013). Risk factors and warning signs. American Foundation for Suicide Prevention.

Aggarwal, C. C. and Zhai, C. (2012). Mining Text Data. Springer, 1 edition.

Bailey, R. K., Patel, T. C., Avenido, J., Patel, M., Jaleel, M., Barker, N. C., Khan, J. A., All, S., and Jabeen, S. (2011). Suicide: current trends. Journal of the National Medical Association, 103(7):614-617.

Burnap, P., Colombo, G., Amery, R., Hodorog, A., and Scourfield, J. (2017). Multiclass machine classification of suicide-related communication on twitter. Online social networks and media, 2:32-44.

De Choudhury, M., Gamon, M., Counts, S., and Horvitz, E. (2013). Predicting depression via social media. In Seventh international AAAI conference on weblogs and social media.

Desmet, B. and Hoste, V. (2014). Recognising suicidal messages in dutch social media. In 9th international conference on language resources and evaluation (LREC), pages 830-835.

Go, A., Huang, L., and Bhayani, R. (2009). Twitter sentiment analysis. Entropy, 17:252.

Gunn, J. F. and Lester, D. (2015). Twitter postings and suicide: An analysis of the postings of a fatal suicide in the 24 hours prior to death. Suicidologi, 17(3).

Jashinsky, J., Burton, S. H., Hanson, C. L., West, J., Giraud-Carrier, C., Barnes, M. D., and Argyle, T. (2014). Tracking suicide risk factors through twitter in the us. Crisis.

Kailasam, V. and Samuels, E. (2015). Can social media help mental health practitioners prevent suicides? Current Psychiatry, 14(2):37-51.

McCarthy, M. J. (2010). Internet monitoring of suicide risk in the population. Journal of affective disorders, 122(3):277-279. 
Miner, G., Elder, J., Hill, T., Nisbet, R., Delen, D., and Fast, A. (2012). Practical Text Mining and Statistical Analysis for Non-structured Text Data Applications. Academic Press, 1 edition.

Moreno, M. A., Jelenchick, L. A., Egan, K. G., Cox, E., Young, H., Gannon, K. E., and Becker, T. (2011). Feeling bad on facebook: depression disclosures by college students on a social networking site. Depression and anxiety, 28(6):447-455.

O’Dea, B., Wan, S., Batterham, P. J., Calear, A. L., Paris, C., and Christensen, H. (2015). Detecting suicidality on twitter. Internet Interventions, 2(2):183-188.

OMS (2014). Preventing suicide: A global imperative. World Health Organization.

Rossi, R. G. (2016). Classificação automática de textos por meio de aprendizado de máquina baseado em redes. $\mathrm{PhD}$ thesis, Universidade de São Paulo.

Rossi, R. G., Lopes, A. A., and Rezende, S. O. (2016). Optimization and label propagation in bipartite heterogeneous networks to improve transductive classification of texts. Information Processing and Management, 52(2):217-257.

Rossi, R. G., Lopes, A. d. A., Faleiros, T. d. P., and Rezende, S. O. (2014). Inductive model generation for text classification using a bipartite heterogeneous network. Journal of Computer Science and Technology, 29(3):361-375.

Sinoara, R. A., Antunes, J., and Rezende, S. O. (2017). Text mining and semantics: a systematic mapping study. Journal of the Brazilian Computer Society, 23(9):1-20.

Sueki, H. (2015). The association of suicide-related twitter use with suicidal behaviour: a cross-sectional study of young internet users in japan. Journal of affective disorders, 170:155-160.

Witten, I. H. and Frank, E. (2005). Data Mining: Practical Machine Learning Tools and Techniques. Elsevier, 2 edition. 